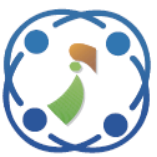

\title{
Epileptic EEG Signal Classification Using Convolutional Neural Network Based on Multi-Segment of EEG Signal
}

\author{
Irwan Budi Santoso ${ }^{1,2} \quad$ Yudhi Adrianto $^{3} \quad$ Anggraini Dwi Sensusiati $^{3}$ \\ Diah Puspito Wulandari ${ }^{1}$ I Ketut Eddy Purnama ${ }^{1 *}$
}

\author{
${ }^{1}$ Department of Electrical Engineering, Institut Teknologi Sepuluh Nopember, Surabaya, Indonesia \\ ${ }^{2}$ Department of Informatics Engineering, Universitas Islam Negeri Maulana Malik Ibrahim, Malang, Indonesia \\ ${ }^{3}$ Faculty of Medicine, Universitas Airlangga, Surabaya, Indonesia \\ * Corresponding author's Email: ketut@te.its.ac.id
}

\begin{abstract}
High performance in the epileptic electroencephalogram (EEG) signal classification is an important step in diagnosing epilepsy. Furthermore, this classification is carried out to determine whether the EEG signal from a person's examination results is categorized as an epileptic signal or not (healthy). Several automated techniques have been proposed to assist neurologists in classifying these signals. In general, these techniques have yielded a high average accuracy in classification, but the performance still needs to be improved. Therefore, we propose a convolutional neural network based on multi-segment of EEG signals to classify epileptic EEG signals. This method is built to overcome data limitations in the convolutional neural network training process and add the ensemble combination process. The multi-segment of EEG signal is formed by splitting the signal without overlapping each channel and converting it into the spectrogram image based on the short-time Fourier transform value. The spectrogram image is then used as input for the convolutional neural network in in-depth training and testing. The convolutional neural network model of the training results is used to classify each EEG signal segment on each test channel before entering the ensemble combination stage for the final classification. To evaluate the performance of our proposed method, we used the Bonn EEG dataset. The dataset consists of five EEG records labelled as A, B, C, D, and E. The experiments on several datasets (AB-C, AB-D, AB-E, AB-CD, AB-CDE, and AB-CD-E) which were arranged from the dataset showed that our proposed method (with segment) performs better than without segment. Our proposed method yielded the best average of classification accuracy which is $99.33 \%, 100 \%, 100 \%, 99.5 \%, 99.8 \%$, and $99.4 \%$ for the $\mathrm{AB}-\mathrm{C}$, AB-D, AB-E, AB-CD, AB-CDE, and AB-CD-E. By these results, the proposed method can outperform several other methods on the same dataset.
\end{abstract}

Keywords: Electroencephalogram, Segment, Short time fourier transform, Spectrogram image, Convolutional neural network, Ensemble combination.

\section{Introduction}

Epilepsy is a chronic brain disease characterized by repeated seizures and involuntary movements involving part or all of the body [1]. Examination of a patient using the EEG does not always result in a precise diagnosis. Some patients were diagnosed to be in normal condition by EEG examination, but they had epilepsy [2]. This mistake can be caused by manual diagnosis by an expert by merely looking at the EEG recordings. Therefore, it is necessary to develop a method for the classification of epileptic EEG signals as a part of diagnostics that has highperformance. Many previous studies had developed these methods, which are generally grouped into two main stages: the signal features extraction and classification of epilepsy with the input of these features $[3,4]$. In theory and practice, these stages have contributed significantly to improve the EEG signal-based epilepsy classification performance. The contribution of the method in the EEG signal features extraction includes the time domain as presented in [5,6], the frequency domain as shown in 
[7-9], the time-frequency domain as presented in [1012], and the domain graph as presented in [13]. Meanwhile, the development of the method of classification/detection of epilepsy signals is relatively less than the feature extraction method. The proposed method for EEG signal features extraction is usually evaluated using public datasets. Among the epileptic EEG signal datasets used as a standard of testing is the epileptic EEG signals dataset from Bonn University, Germany [14]. Several studies have used this dataset, including by researchers in [9-11,15-22].

From several previous studies, the average performance of their proposed method, in this case, was high, but the performance still needed to be improved. From a machine learning perspective, efforts to improve performance are still constrained by the limited data available. Besides, most of the researchers focused on getting the best method to extract epileptic EEG signal features. Therefore, there are many variations of signal features extraction methods but rarely focus on improving classification performance by modifying the classifier or focus on both. In time-domain studies, they focus more on how to get a signal pattern that represents the original signal with the least possible noise. However, in the frequency domain, they focus more on how to get a method to obtain the frequency's main features. Since the EEG signal is a data series, using only the time or frequency domain is not enough, so the EEG signal must be processed in the time-frequency domain, for example, using short-time Fourier transform (STFT) [20], empirical mode decomposition (EMD) [10], or wavelet transform $[16,17]$. However, the extraction of features in this domain does not always result in the best classification performance. There is no guarantee that a classifier can work properly with these features because the features extraction process does not involve the classifier itself. Therefore, additional steps are needed to solve this problem.

In this paper, we proposed a method to enrich features and select the features by involving the classifier. This method is done by splitting the EEG signal into several segments (multi-segment) and converting it into the spectrogram image [23-25] and involving the convolutional neural network (CNN) [26] as the classifier. The multi-segment of EEG signal is created to enrich the training's data, thereby strengthening the classification performance. In contrast, the reasons for using a spectrogram image for each EEG signal segment are: first, the spectrogram image is a visual representation of the signal in the form of the image [23]. The spectrum frequency in the image spectrogram varies from time to time, while the different colors in the image represent different energy values. Second, it contains more unknown EEG signal features and has better performance on CNN [26]. In this paper, the spectrogram image of the signal obtained from the STFT value is mapped to the RGB colormap [24, 25]. The third reason, visually with the image spectrogram, there is a clear difference between epileptic and nonepileptic EEG signals, as showed in Fig. 1. STFT is chosen to determine the spectrogram determination because of its ability to calculate complex amplitude over time and frequency on non-stationary EEG signals [27]. Simultaneously, the CNN method was chosen as a method for the classification of epileptic EEG signals because it has a deep learning algorithm that can select features optimally based on the loss function to achieve high performance in classifying images [26].

There are four main stages of the proposed method for the classification of epileptic EEG signals. The first step is determining the multi-segment of the EEG signal. Second is determining the STFT value for each segment of the EEG signal with the same input parameters for each segment and label (class) the scenario. Third is creating the spectrogram image by mapping the STFT value to the RGB color map for each signal segment. The final step is classifying each signal segment with $\mathrm{CNN}$ and the ensemble of the CNN results. In the classification of epileptic EEG signals with a spectrogram image value input, CNN's training is done based on the $\mathrm{CNN}$ architecture.

The contributions of this paper are as follows:

- Forming the multi-segment and spectrogram image for each EEG signal to enrich the features. Experiments on the epileptic EEG dataset from the University of Bonn have shown that splitting the EEG signal into several segments (multisegment) and converting it to the spectrogram image gives $\mathrm{CNN}$ a significant increase in classification.

- Establishing an ensemble method for the classification results of each EEG signal segment with CNN. This method is used to perform the final classification and to provide improved classification performance in testing.

In this paper, Section 2 discusses the related works about the classification of the epileptic EEG signal. Section 3 describes the materials and methods. In Sections 4 and 5, we intensively discuss the experiments and the results. Lastly, Section 6 contains the conclusions of this study.

\section{Related work}

In this study, we used the Bonn EEG dataset to evaluate the proposed method. Therefore, in this 
section, we discuss several recent studies that use these datasets. The studies were based on two methods namely conventional methods and CNN.

The studies were based on conventional methods, such as those conducted by Alçin et al [21], Tiwari et al. [28], X. Zhao et al. [29], Sharma et al. [30], and Gupta et al. [31]. Alçin et al. [21] converted the EEG signal into a spectrogram based on the value of STFT. Grey level co-occurrence matrix (GLCM) was used for feature extraction and passed to the fisher vector (FV) encoder before the classification stage. The test results using the extreme learning machine (ELM) classifier for five classes obtained an accuracy of 96.4\%. This performance indicates that the spectrogram, GLCM, and FV successfully extract important signal features for the five-class classification.

Tiwari et al. [28] used the pyramid of the Difference of Gaussian (DoG) for signal key-point detection. Local binary patterns (LBP) are computed at key points and determined a histogram as a feature set. Classification using SVM on the AB-E and ABCD-E datasets obtained an accuracy of $100 \%$ and $98.8 \%$ respectively. The same case was carried out by $X$. Zhao et al. [29]. They measured that the instantaneous energy changes in the EEG signal to obtain features. EEG signal was classified using several classifiers. The combination of instantaneous energy-based features with back propagation neural network (BPNN) on the AB-E and AB-CD-E dataset yielded an accuracy of $100 \%$ and $99.1 \%$ respectively. The result shown by Tiwari et al. [28] is the success in finding the key-point of the signal, whereas $X$. Zhao et al. [29] get better results by considering several classifiers.

Sharma et al. [30] used iterative filtering (IF) to decompose the signal into intrinsic mode functions (IMFs). The signal features were taken from the IMF function and the amplitude envelope AE included the k-nearest neighbor entropy estimator (KNNE), log energy entropy (LEE), shannon entropy (SE), and poincar'e plot parameters. Experiments on the ABCD-E dataset using the random forest classifier obtained an accuracy of $98 \%$. Signal decomposition was also carried out by Gupta et al. [31] who proposed a multirate filter bank structure to decompose the signal into brain rhythms and model it with fractional Brownian motion $(\mathrm{fBm})$ and fractional Gaussian noises (fGn). The hurst exponent and autoregressive moving average (ARMA) parameters were features of the EEG signal. The test results using the binary SVM classifier on the AB$\mathrm{CD}$ and $\mathrm{AB}-\mathrm{E}$ datasets were obtained an accuracy of $97.7 \%$ and $97.27 \%$ respectively. The main problem of this method is that many processes and the more tuning of parameters to extract the features. Therefore, limited data in the experiment cause the classification result not optimal.

Several recent studies on the classification of epileptic EEG signals based on the CNN include studies conducted by Ullah et al. [32], W. Zhao et al. [33], Akut [34], and Tu"rk and Ozerdem [35]. Ullah et al. [32] divided the EEG signal into several overlapping sub-signals. EEG signal classification was obtained by inputting the raw sub-signal dataset into the pyramidal one-dimensional convolutional neural network (P-1D-CNN) models (14 layers). From the experiment using the method on the ABCD-E dataset, it obtained an average of accuracy which is $99.1 \%$. Similar study was also conducted by W. Zhao et al. [33]. They divided each EEG signal channel into 23 sub-signals and proposed 1D CNN for detection of epileptic seizures. The results of testing the model on the Bonn dataset obtained an accuracy of $97.63 \%-99.52 \%$ for the two-class and $96.73 \%-98.06 \%$ for three classes.

Another study was conducted by Akut [34], which divided the EEG signal into 5 main subbands and used a discrete wavelet transform (DWT) to extract the lowest frequency and eliminate the highest frequency. Training using CNN (23 layers) showed that the model works properly on small datasets. Classification using this model on the AB-CD-E dataset yielded an accuracy of $99.4 \%$. Preprocessing of EEG signals was also conducted by Türk and Ozerdem [35]. They formed the scalogram using a continuous wavelet transform (CWT) on each signal channel and resized the image. The testing results using CNN (5 layers) on the A-E and A-D-E datasets obtained an accuracy of $99.50 \%$ and $99 \%$ respectively. The main problem of their proposed method is that resizing the image has an impact on classification.

Next is considering previous studies by using conventional methods. Many methods have been developed to obtain EEG signal features. On the other hand, the best performance in the classification /detection of epilepsy is not necessarily obtained by using these features. Therefore, researchers often involve several methods by tuning parameters to get the features and use one or several classifiers. This approach is certainly ineffective and time-consuming. Meanwhile, the CNN-based epileptic EEG signal classification/detection in this case provides a solution to the problem of conventional methods. However, several things must be considered, including limited data availability, preprocessing of the EEG signal and the CNN architecture. Studies by Ullah et al. [32] and W. Zhao et al. [33] split the original signal to overcome data limitations. The raw 
data resulted from the splitting (1D) were directly forwarded into CNN. Compared to Akut [34] without signal splitting but processing the signal with DWT (2D) before implementation to CNN gives better results than Ullah et al. [32] for the same dataset. On the other hand, overcoming data limitations must be done to improve CNN performance. Another case conducted by Türk and Ozerdem [35] although using a scalogram (2D) but resizing image affects the classification performance. This paper proposes a methodology involving signal splitting (segment), signal preprocessing (2D), CNN, and ensemble in the classification of epileptic EEG signals. Our proposed method differs from existing method in several aspects: (i) segments formed from the original EEG signal without overlapping are to keep mutually exclusive characteristics (ii) adding preprocessing to form a spectrogram image (2D) (iii) using a single CNN model (iv) classifying each testing segment using the same CNN model, before deciding on the classification results with the ensemble combination. For the experiment, this paper focuses on classifying normal (healthy) (AB) EEG signals with epilepsy (C, D, E, CD, CDE, CDE). Therefore, there are six scenarios for evaluation.

\section{Material and methods}

This section further explains the dataset and the proposed method stages for the classification of epileptic EEG signals. The dataset used in the experiment is the dataset of epileptic EEG signals taken from Bonn University, Germany. The proposed method for the epileptic EEG signals classification includes several main stages as showed in Fig. 2.

\subsection{Dataset of experiment}

The dataset used in the experiment is the epileptic EEG signals dataset available in [14] and described by [36]. The dataset consists of Set A-Set E. Each dataset contains 100 single-channel EEG signals and it is recorded for 23.6 seconds. These signals were selected after visual inspection of the artifacts caused by the movement of the eye muscles. Set A and Set $B$ were EEG signal data obtained from five healthy volunteers with their eyes open and closed. Sets C, D, and $\mathrm{E}$ were the EEG signals obtained from five people with epilepsy patients at their preoperative diagnosis. Set $\mathrm{C}$ and $\mathrm{D}$ signals were obtained at seizure-free intervals (inter-ictal), while Set E was obtained at seizure (ictal). The Set $\mathrm{C}$ signal was obtained by placing the electrode opposite to the epileptogenic zone, while the Set D and $\mathrm{E}$ were in the epileptogenic zone.

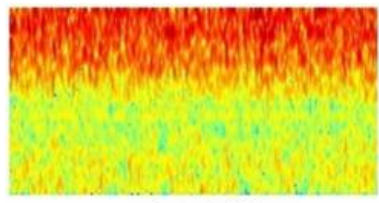

Set A(4)

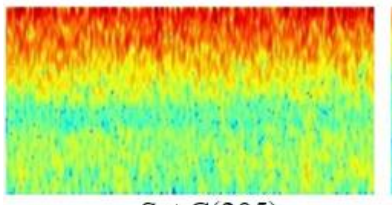

Set C(205)

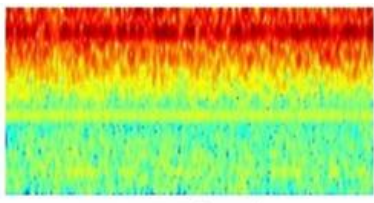

Set $B(110)$

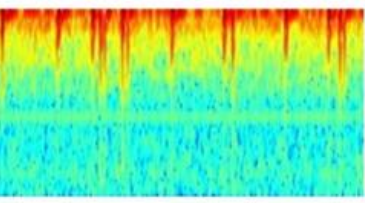

Set D(310)

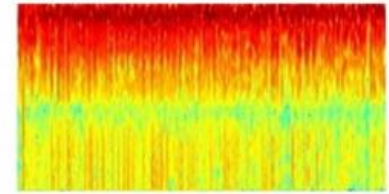

Set $\mathrm{E}(405)$

Figure. 1 Example of spectrogram images of epileptic EEG signal taken from the Bonn EEG dataset

From the description of the dataset, we arrange several scenarios of the experimental dataset. This study's dataset scenario is focused on the classification of EGG signals in epileptic patients and healthy people with several combinations among the existing datasets. Six datasets are created from five datasets (Set A-Set B) to evaluate the proposed method's performance. The description of the six datasets can be seen in Table 1 .

\subsection{Segment of EEG signal}

Epileptic EEG signals are periodic, nonstationary, and their values vary from time to time. The representative signal value of a certain period (quasi-stationary) in this study is obtained by dividing the EEG signal of each channel of each class into smaller and mutually exclusive segments $[9,37]$. Each segment consists of EEG signal data in a certain time window, for example in one of the cases in this study each segment has a time window of $4.72 \mathrm{~s}$, as shown in Fig. 3. A segment's formation on the signal is used to get many features and to enrich data in training or testing.

Fig. 3 is an example of determining the segment of an EEG signal from the Bonn EEG database. In this example, each EEG channel's signal is divided into five segments, with each segment containing the signal data for $4.72 \mathrm{~s}$. Since each channel contains 4097 data points of 23.6s, the size of segment 1 is 819 points, segment 2 is 819 points, segment 3 is 819 points, segment 4 is 819 points, and segment 5 is 821 points. 


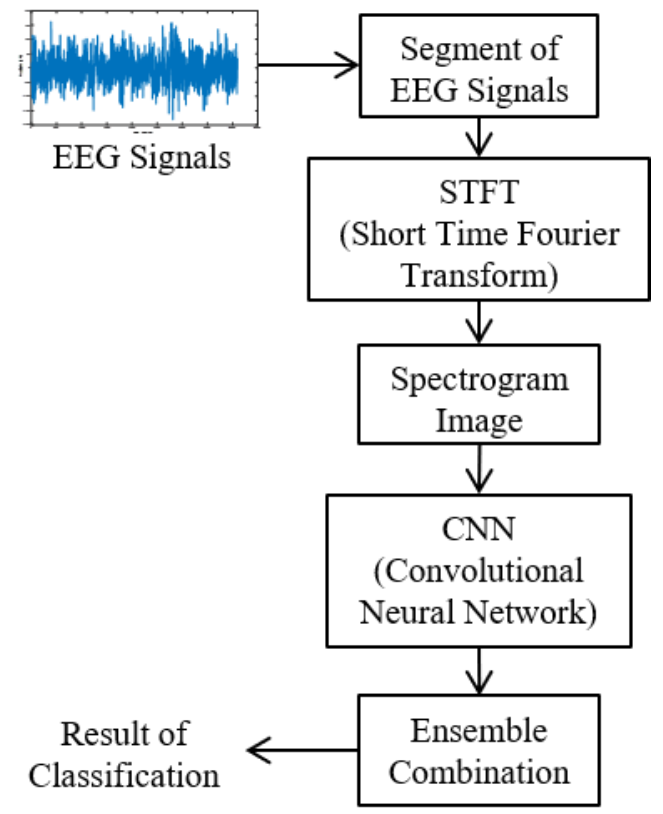

Figure. 2 The stages of the proposed method for epileptic EEG signals classification

\subsection{Short-time fourier transform}

Short-time Fourier transform (STFT) is implemented to transform the signal from the time domain to the time-frequency domain. This method is used to analyze the smallest part of the EEG signal in time using windowing [38]. Mathematically, the definition of STFT can be written as follows

$$
\operatorname{STFT}(v, u)=\int_{-\infty}^{\infty} x(t) w(t-u) e^{-j v t} d t
$$

where $x(t)$ is the EEG signal analysed and $w(t)$ is the window with its energy concentrated around $u$. From STFT, the signal spectrogram value is obtained with the following equation

$$
S=|\operatorname{STFT}(v, u)|^{2}
$$

The spectrogram values measure the amount of energy around the time-frequency $(v, u)$. In this study, STFT is obtained by a discrete approach $[24,39]$, which can be written as follows

$$
\operatorname{STFT}(v, u)=\sum_{t=0}^{L-1} x(t) w(t-u) e^{-j 2 \pi v t / L}
$$

where $w(t)$ is the window function on the $L$-point. For each window, the Fourier Transform process is calculated by using the Discrete Fourier Transform (DFT). Furthermore, the amplitude spectrum obtained from the STFT is converted to decibel $(\mathrm{dB})$.

The windowing technique for STFT in this study is the Blackman window defined in [40]. This technique is chosen based on the characteristic of the
Table 1. Dataset scenarios for epileptic EEG signal classification

\begin{tabular}{clrlc}
\hline No & Datasets & \multicolumn{3}{c}{ Description } \\
\cline { 3 - 5 } & & Class 1 & \multicolumn{1}{c}{ Class 2 } & Class 3 \\
\hline 1 & AB-C & Healthy & Inter-ictal & - \\
2 & AB-D & Healthy & Inter-ictal & - \\
3 & AB-E & Healthy & Ictal & - \\
4 & AB-CD & Healthy & Inter-ictal & - \\
5 & AB-CDE & Healthy & Epilepsy & - \\
6 & AB-CD-E & Healthy & Inter-ictal & Ictal \\
\hline
\end{tabular}

epileptic EEG signal and the assumptions on DFT. Implementation of DFT in STFT assumes a periodic extension of the input vector. Therefore, the suitable windowing technique used in this case is the periodic Blackman window.

\subsection{Spectrogram image}

A spectrogram image is obtained by changing the $\mathrm{S}$ index in the previous step into RGB. This change in the spectrogram image represents the frequency amplitude $[24,25]$ or the frequency spectrum. The steps to change $S$ to RGB are explained as follows:

1. Rescale the $S$ value in the range $[0,1]$.

2. Change the $S$ value in the range $[0,1]$ into the range $[0,255]$.

3. Change the $S$ index into the RGB color map by using a colormap jet.

The jet colormap is a variant of HSV. The color map starts with dark blue, ranges through shades of blue, cyan, green, yellow, and red, and ends with a deep red. In this study, the spectrogram image is a visual representation of the frequency spectrum so that a lot of information can be retrieved [23]. Besides, the CNN classifier will provide high performance when working on RGB image objects [41]. Fig. 4 is an example of the EEG signal of healthy people and epileptics, which is split into five segments then the spectrogram image is determined in each segment.

\subsection{Convolutional neural network}

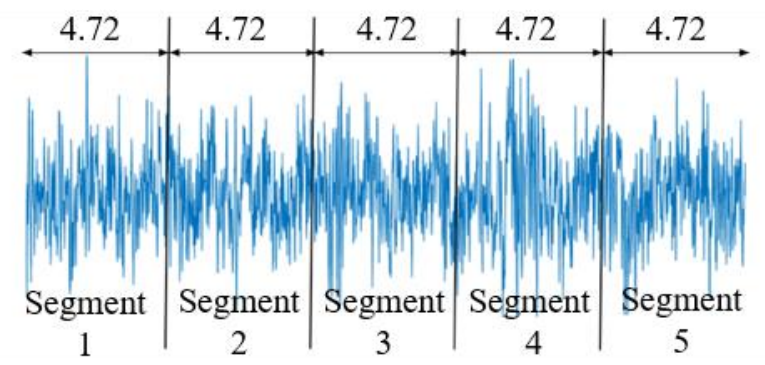

Figure. 3 Example of the segment of an EEG signal 


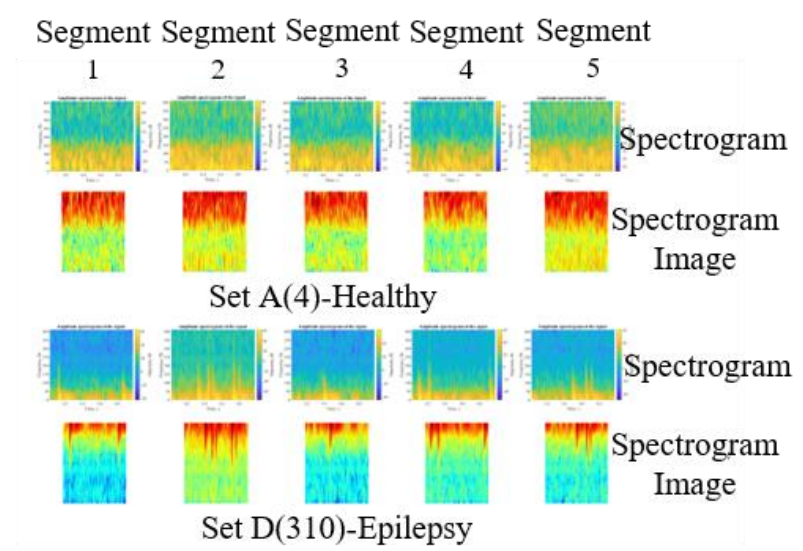

Figure. 4 Example of the spectrogram images for each segment on a signal channel in dataset A (healthy) and D (epilepsy)

Convolutional neural network $(\mathrm{CNN})$ is a type of feed-forwarding neural networking applied to visual images and has a high performance $[42,43]$. In this study, the CNN architecture includes several layers, including the input layer, convolutional layer, batch normalization layer, activation layer, pooling layer, fully connected layer, and output (classification) layer. Fig. 5 is the $\mathrm{CNN}$ architecture used in the epileptic EEG signals classification with these layers.

\subsubsection{Input layer}

The input layer is the layer for inserting images into the network and performing data normalization. In this study, the input is a spectrogram image in RGB, so that there are three channels in a multispectral or hyperspectral image. The spectrogram image size depends on the spectrogram size of the STFT results (e.g. width=a height=b). While data normalization is obtained by subtracting each input image with the image's mean value [43]. It is written $\hat{Z}=Z-\bar{Z}$ where $\hat{Z}$ is the normalized value of the spectrogram image, $Z$ is the value of the spectrogram image, and $\bar{Z}$ the average value of spectrogram image.

\subsubsection{Convolutional layer}

The convolutional layer is a layer that will convolute the input data (spectrogram image) or from the previous layer by shifting a filter to produce the feature map. The convolution process will yield many feature maps to butter understand the characteristics of the spectrogram image $[44,45]$.The convolution operation can be written as follows

$$
\tilde{Z}=f(W \hat{Z}+b)
$$

with $\tilde{Z}$ is the output of the convolution process, $f($. is the activation function, $W$ is the weights, and $b$ is the bias. The weight on the convolutional layer will experience an update process to improve the image classification results in the training process. The update process in training is done on all weights in each convolutional layer. A set of weights applied to a region in an image is called the filter. In this study, the filter or kernel used refers to [46] with the following equation

$$
W_{i j} \sim U\left[-\frac{1}{\sqrt{n}}, \frac{1}{\sqrt{n}}\right]
$$

where $U\left[-\frac{1}{\sqrt{n}}, \frac{1}{\sqrt{n}}\right]$ as a uniform distribution with interval parameters $\left(-\frac{1}{\sqrt{n}}, \frac{1}{\sqrt{n}}\right)$ and $n$ is the size of the previous layer (number of columns $W$ ). In this study, the number of filters used for the first layer is 30 , the second is 60 , and the third is 120 , while the filter size is $5 \times 5$ for each layer. In the convolution process, stride $(s)$ and padding $(p)$ must be determined [47]. The stride and padding used in the convolution process are one (1) and the same padding as shown in Fig. 5.

\subsubsection{Batch normalization layer}

The batch normalization layer is normalizing each input channel in mini-batch. This process is needed to speed up training on CNN and to reduce the network initialization sensitivity. To achieve the results, the batch normalization layer is placed between the convolutional and ReLU as shown in Fig. 5. In this study, the batch normalization process refers to reference [48]. The first layer of the process is to normalize each channel's activation by subtracting each channel with the mini-batch average and dividing by the mini-batch standard deviation. Then, the layer shifts the input and scales it by the scale factor.

\subsubsection{Activation layer}

The activation layer applies an unsaturated activation function to enhance the nonlinear nature of the decision function. In this study, the activation function used is the rectified linear unit (ReLU) [41, 44], which is presented in the following equation

$$
\tilde{Z}_{R}(\hat{F})=\left\{\begin{array}{l}
\hat{F}, \hat{F} \geq 0 \\
0, \hat{F}<0
\end{array}\right.
$$

with $\hat{F}$ is the output of the convolution process, which has entered the mini-batch process. 


\subsubsection{Pooling layer}

The pooling layer usually follows a convolutional layer and is a non-linear sampling method. The pooling process is used to reduce the spatial size of the representation. This process also reduces the number of calculations and controls overfitting. In this study, the pooling used is max pooling, which partitions the input layer into a rectangular set with each region's maximum output [41,49]. The filter size used by the pooling layer is $4 \times 4$ with a stride size of 2 , as shown in Fig. 5.

\subsubsection{Fully connected layer}

The fully connected layer is used after the convolutional layer and max-pooling layer. In this layer, the feeds back process is done by refining the previous layer's weight and bias. This process also reduces the loss of feature information. The results of this layer forward the output layer for classification [41].

\subsubsection{Output (classification) layer}

The output (classification) layer functions to show the classification results, namely, accuracy and loss. The loss is the deviation between the predicted and the target labels. The two of most widely used activation functions for classification are softmax and sigmoid [41]. In this study, the activation function used in the output layer is softmax [50], as in the following equation

$$
y_{k}\left(Z^{*}\right)=\frac{\exp \left(z_{k}^{*}\right)}{\sum_{j=1}^{C} \exp \left(z_{j}^{*}\right)}, k=1, \ldots, C
$$

where $y_{k}$ is the softmax output in the $k$-class, $Z^{*}$ is the output of the fully connected layer process in the $k$-class, and $C$ is the number of classes (labels).

\subsection{Ensemble combination}

The ensemble method used in this study is almost similar to the bagging method [51]. The ensemble method is only applied at the testing and only uses one CNN model training results to classify each segment on the testing dataset. Fig. 6 shows how the process of training and testing the EEG signal dataset by applying an ensemble combination of the classification results of each segment with $\mathrm{CNN}$. The ensemble combination used in determining the output is simple majority voting as in the bagging method. Based on the output layer at the CNN training stage, the classification results for each segment of the testing dataset can be determined by using the equation as follows

$$
h_{i}=\arg \max _{k}\left(y_{i k}\right), i=1,2, \ldots, r ; k=1, \ldots, C
$$

with $r$ is the number of EEG signal segment, and $C$ is the number of label (class). If $v_{i, k}$ is the result of voting with the determination $v_{i, k}=1$, the evaluation result is the same as the actual class and 0 if it is not the same, then the total voting in the ensemble can be determined by using the equation as follows

$$
V_{k}=\sum_{i=1}^{r} v_{i k}, k=1, \ldots, C
$$

then the ensemble results can be obtained by using the equation as follows

$$
E C=\arg \max _{k}\left(V_{k}\right), k=1, \ldots, C
$$

\subsection{Cross validation and performance evaluation}

To avoid the possibility of overfitting and obtain reliable performance from the $\mathrm{CNN}$ model, we applied the k-fold cross-validation technique [52] for each scenario. In this study, we use is 5-fold crossvalidation. The entire dataset is randomly split into five folds with the same sample size. For each fold, four subsets are for training, and the rest for testing. The process is repeated five times. Each test dataset is obtained five performances in the classification, and this performance average is the last performance. In general, the performance of the proposed method in epileptic EEG signal classification is evaluated by statistical measures of sensitivity (SEN), specificity (SPE), and accuracy (ACC) [53], which are defined as follows.

$$
\begin{gathered}
S E N=\frac{T P}{T P+F N} 100 \% \\
S P E=\frac{T N}{T N+F P} 100 \% \\
A C C=\frac{T P+T N}{T P+T N+F P+F N} 100 \%
\end{gathered}
$$

where $T P$ and $T N$ are the correct total numbers in the classification of the EEG signal of healthy people and the correct total number in the classification of the EEG signal of patients with epilepsy, $F P$ and $F N$ are the total numbers that are wrong in the classification of the EEG signal of healthy people and the total number that is wrong in the classification of the EEG signal of people with epilepsy. 


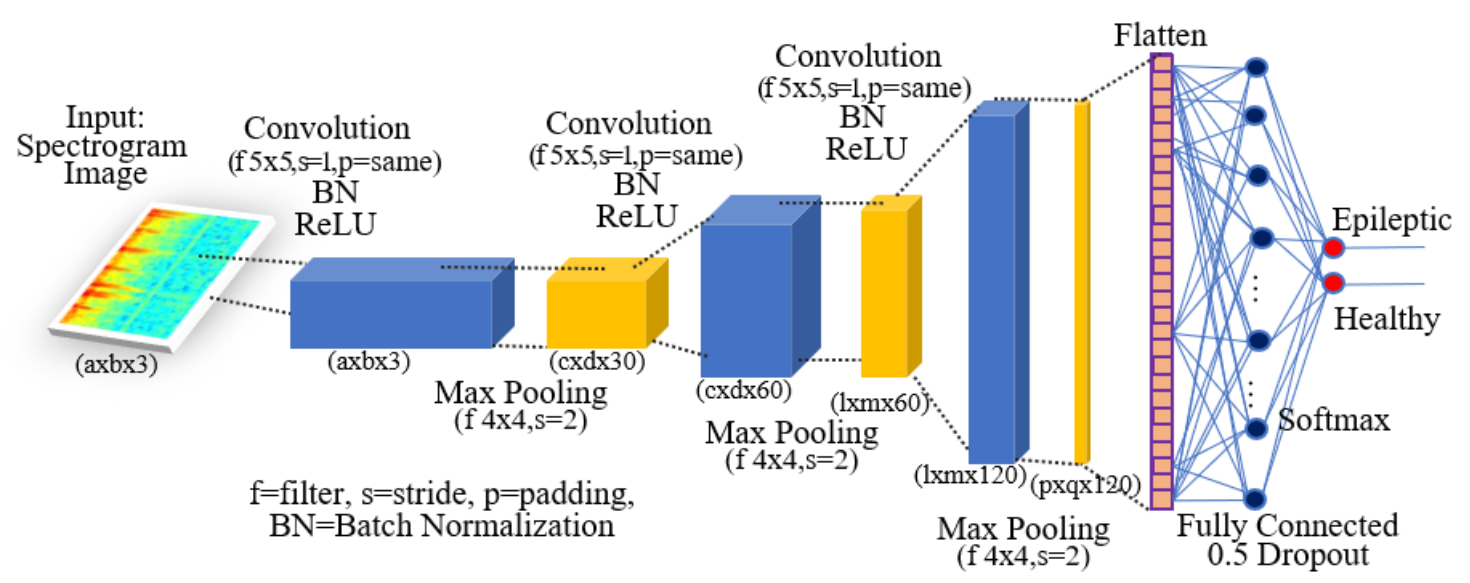

Figure. 5 The architecture of CNN for epileptic EEG signals classification

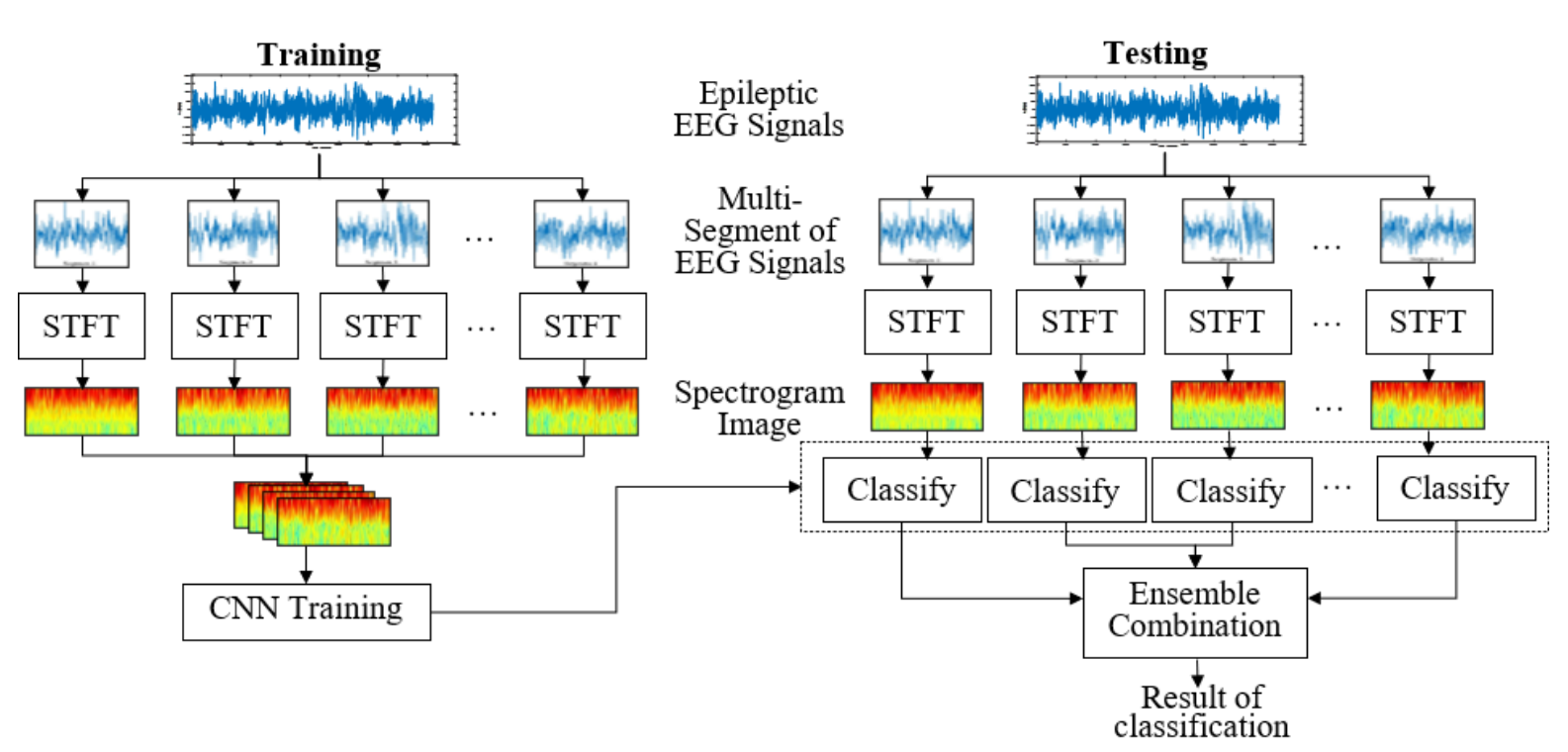

Figure. 6 Training and testing using $\mathrm{CNN}$ and Ensemble Combination

\section{Experiment}

In this section, we use the epileptic EEG signal dataset to evaluate the proposed method's performance. The evaluation is carried out by referring to the six experimental dataset scenarios as in the previous chapter. Experiments were implemented using MATLAB with detailed experimental settings for each EEG dataset scenario as follows.

\subsection{Data preparation}

Although the Bonn dataset has been widely used, we use it in this study which is different from many previous studies. Apart from being different from the proposed method, this study focuses on the epileptic EEG signals classification with scenarios, as shown in Table 1. To evaluate the proposed method's performance on six datasets arranged from five sets of EEG signals, it is necessary to specify the number of training and testing datasets for each scenario. In this study, we use 5-fold cross-validation so that the composition of the training and testing dataset is $80 \%$ and $20 \%$ as shown in Table 2. The AB-C, AB$\mathrm{D}$, and $\mathrm{AB}-\mathrm{E}$ datasets have the same number of training and testing samples. Besides, for $\mathrm{AB}-\mathrm{CDE}$ and $\mathrm{AB}-\mathrm{CD}-\mathrm{E}$ also has the same number of samples of 400 and 100. Even though each experimental fold in one dataset has the same number of training and testing samples, the composition of the number of samples for each class/label can be different/varied. From this dataset, the proposed method is implemented in each fold. Thus, five classification performance of epileptic EEG signal is obtained for each dataset. The proposed method's performance is obtained by calculating the average of the five performances for each dataset. 


\subsection{Parameters settings}

Based on the proposed method's steps, each channel of the EEG signal is split into several smaller segments. In this study, the experiment is done with the number of segments $(r)$ as different and the best selected based on the resulting accuracy value. The number of segments $(r)$ used for testing includes 1 , $3,5,7,9,11$, and 13. Each signal segment is then determined spectrogram by using STFT. In this study, the windowing technique for STFT uses a Blackman window with a window length of 32 , while the Fourier transform process uses FFT with a length of FFT of 256 and the sample rate (Fs) adjusted to the length of the segment is made on each EEG signal channel. Each spectrogram obtained from each segment is converted into a RGB (spectrogram image) in the range $[0,255]$.

For the CNN training process, referring to the CNN architecture in Fig. 5, where the training input is a spectrogram image with dimensions adjusting to the resulting spectrogram image's size. All CNN training for each fold and dataset are performed using "stochastic gradient descent with momentum" (SGDM) to determine the optimal weight [43]. The values of momentum and learning rate used are 0.9 and 0.001. To reduce overfitting, L2 regularization with the parameter used is 0.0001 . The dropout rate is 0.5 used in the fully connected (FC) layer during classification. For all of the training in this study, batch size and a maximum epoch are 128 and 500.

\section{Results and discussion}

In this section, experimental results are generated based on the dataset scenario described in Section 3 and Section 4. The test results using the proposed method in each dataset scenario are begun by splitting each EEG signal channel into $r$ segments.

For $r=1$, it means that the EEG signal channel is still as it came from (without segment). The example, if the AB-C dataset is implemented with 240 and 60 data for training and testing, the training and testing data used remains the same as 240 and 60 . For $r=3$, each EEG signal channel is split into three segments so that the total data training will be three times ( 240 x $3=720$ segment). Likewise, for $r=5, r=7, r=9$, $r=11$, and $r=13$, the total training data will be 5 times ( $240 \times 5=1200$ segments $), 7$ times $(240 \times 7=1680$ segments), 9 times $(240 \times 9=2160$ segments), 11 times $(240 \times 11=2640$ segments $)$ and 13 times (240 $\mathrm{x} 13=3120$ segments). This treatment is also applied to each dataset and fold.

The number of segments on each channel and the dataset scenario will determine the spectrogram's number and spectrogram image. Spectrogram image on each segment as input to CNN training and indirectly enrich training data. The dimensions of the input spectrogram image in each dataset vary depending on the segment's length on each EEG signal channel and the parameters on STFT. The windowing technique of STFT in this study uses the Blackman window with window length $=32$, length of FFT $=256$, and sample rate $(\mathrm{Fs})=$ length of the segment. From these parameters for $r=1$, then Fs $=$ 4097 , and a spectrogram image is obtained based on the STFT value with dimensions of $129 \times 509$. For $r=3, r=5, r=7, r=9, r=11$, and $r=13$, the sample rates used are $365,819,585,455,372$, and 315 respectively and produce a spectrogram image with dimensions as in Table 3. Especially for $r=9$ and $r=11$, we discard the last remaining sample, while for the others, the rest of the sample is merged with the last segment. Table 3 shows the input dimensions of the different spectrogram images on CNN training. The image spectrogram dimensions are different because the number of segments on each EEG signal channel is different, while the parameters for STFT are fixed.

For testing, each EEG signal with a length of 4097 in the test set is divided into $r$ segments without overlapping as for training. Each spectrogram image $\left(Z_{i}, i=1,2, \ldots, r\right)$ obtained from each segment will be forwarded to the same CNN model to be classified. The classification results are then forwarded to the ensemble combination to determine the signals' final classification results. The ensemble combination is used to decide whether an original signal is a seizure signal or not by considering all segments' classification results.

\subsection{Experimental Results}

The results of training with CNN in each dataset and fold scenario showed that the proposed method for each training produced $100 \%$ training accuracy. Furthermore, the test results on each testing dataset and fold of the proposed method can be seen in Table 3 and Table 4.

Using the ensemble combination of $\mathrm{CNN}$ classification results on the $\mathrm{AB}-\mathrm{C}$ dataset obtained the best average performance of classification for the number of segments 7,11, and 13. The average of accuracy, sensitivity, and specificity of the proposed method was $99.33 \%, 99.09$, and $100 \%$. These results are much better than the classification results without performing segment with an average improvement of $4.66 \%(99.33 \%-94.67 \%)$ for accuracy, $1.95 \%$ (99.09\%-97.14\%) for sensitivity and $9.33 \%(100 \%$ $90.67 \%$ ) for specificity. Based on the dispersion of 
Table 2. Samples of training and testing on each fold and scenario

\begin{tabular}{|c|c|c|c|c|c|c|c|c|c|c|c|}
\hline \multicolumn{2}{|l|}{ Datasets } & \multicolumn{2}{|c|}{ Fold-1 } & \multicolumn{2}{|c|}{ Fold-2 } & \multicolumn{2}{|c|}{ Fold-3 } & \multicolumn{2}{|c|}{ Fold-4 } & \multicolumn{2}{|c|}{ Fold-5 } \\
\hline & & Tra & Tes & Tra & Tes & Tra & Tes & Tra & Tes & Tra & Tes \\
\hline \multirow{2}{*}{$\begin{array}{c}\text { AB-C } \\
\text { Tra }=240, \text { Tes }=60\end{array}$} & $\mathrm{AB}$ & 163 & 37 & 157 & 43 & 167 & 33 & 156 & 44 & 157 & 43 \\
\hline & $\mathrm{C}$ & 77 & 23 & 83 & 17 & 73 & 27 & 84 & 16 & 83 & 17 \\
\hline \multirow{2}{*}{$\begin{array}{c}\text { AB-D } \\
\text { Tra }=240, \text { Tes }=60\end{array}$} & $\mathrm{AB}$ & 165 & 35 & 160 & 40 & 159 & 41 & 159 & 41 & 157 & 43 \\
\hline & $\mathrm{D}$ & 75 & 25 & 80 & 20 & 81 & 19 & 81 & 19 & 83 & 17 \\
\hline \multirow{2}{*}{$\begin{array}{c}\text { AB-E } \\
\text { Tra }=240, \text { Tes }=60\end{array}$} & $\mathrm{AB}$ & 158 & 42 & 155 & 45 & 164 & 36 & 161 & 39 & 162 & 38 \\
\hline & $\mathrm{E}$ & 82 & 18 & 85 & 15 & 76 & 24 & 79 & 21 & 78 & 22 \\
\hline \multirow{2}{*}{$\begin{array}{c}\text { AB-CD } \\
\text { Tra }=320, \text { Tes }=80\end{array}$} & $\mathrm{AB}$ & 162 & 38 & 164 & 36 & 154 & 46 & 166 & 34 & 154 & 46 \\
\hline & $\mathrm{CD}$ & 158 & 42 & 156 & 44 & 166 & 34 & 154 & 46 & 166 & 34 \\
\hline \multirow{2}{*}{$\begin{array}{c}\text { AB-CDE } \\
\text { Tra }=400, \text { Tes }=100\end{array}$} & $\mathrm{AB}$ & 163 & 37 & 166 & 34 & 156 & 44 & 155 & 45 & 160 & 40 \\
\hline & $\mathrm{CDE}$ & 237 & 63 & 234 & 66 & 244 & 56 & 245 & 55 & 240 & 60 \\
\hline \multirow{3}{*}{$\begin{array}{c}\text { AB-CD-E } \\
\text { Tra }=400, \text { Tes }=100\end{array}$} & $\mathrm{AB}$ & 156 & 44 & 157 & 43 & 166 & 34 & 162 & 38 & 159 & 41 \\
\hline & $\mathrm{CD}$ & 163 & 37 & 166 & 34 & 158 & 42 & 156 & 44 & 157 & 43 \\
\hline & $E$ & 81 & 19 & 77 & 23 & 76 & 24 & 82 & 18 & 84 & 16 \\
\hline
\end{tabular}

*)Tra=training,Tes=testing

accuracy, each fold's performance in the number of segments 7, 11, and 13 has an accuracy value of $98.33-100 \%$, sensitivity $97.73-100 \%$, and specificity of $100-100 \%$. In contrast, the dispersion of accuracy, sensitivity, and specificity for without segment is 91.67-96.67\%, 93.18-100\%, and 87.50-94.12\%. From these dispersion values, accuracy dispersion for with segment is much better than the dispersion without segment. By considering the performance value and the number of signal segments formed, the test on the dataset shows that the proposed method for the number of segments of 7 gives the best results.

For testing on the AB-D dataset, in general, the proposed method's performance based on multisegment of the signal is also much better than the performance without the segment of the signal. The best results are indicated by an average value of $100 \%$ accuracy for the number of segments 5-13 so that the average sensitivity and specificity are also $100 \%$. In contrast, without segment, the average of accuracy, sensitivity, specificity was $97.67 \%$, $98.14 \%$, and $95.10 \%$. From this value, it can be determined that the average performance improvement of the method by forming the segment of the signal was $2.33 \%$ for accuracy, $1.22 \%$ for sensitivity, and $4.9 \%$ for specificity.

The same results were also obtained in the AB-E dataset. The performance of the proposed method was better than without the segment of the signal. The best results were obtained in the number of segments 7 and 9 with $100 \%$ accuracy, sensitivity, and specificity values. Meanwhile, the proposed method's average improvement for accuracy, sensitivity, and specificity was $2.67 \%, 2.95 \%$, and $2.3 \%$. Likewise, in the $\mathrm{AB}-\mathrm{CD}$ dataset, the best performance was obtained from the proposed method in the number of segments 9 and 11 with the average values of accuracy, sensitivity, and specificity in the classification of $99.5 \%, 99.03 \%$, and $100 \%$. The average improvement given by the proposed method for accuracy, precision, and specificity was $2.25 \%$, $1.42 \%$, and $3.14 \%$.

Overall, for testing on the AB-CDE dataset, it also provides better classification performance than the implementation without the segment of the original signal. The methods proposed implemented in the number of segments 11 and 13 have the same and best performance compared to the others with an average accuracy, sensitivity, and specificity of $99.8 \%, 99.48 \%$, and $100 \%$, respectively. The average improvement of the proposed method with the signal segment was $3 \%$ for accuracy, $5.28 \%$ for sensitivity, and $1.31 \%$ for specificity. The dispersion for accuracy, sensitivity, and specificity of the proposed method was $99-100 \%, 97.37-100 \%$, and $100-100 \%$. From this dispersion, it is identified that the proposed method has high performance and consistency.

In the AB-CD-E dataset, the best performance value is also dominated by the proposed method by forming the signal segment. The best results were obtained in the number of segments 9 with an average accuracy of $99.4 \%$. The average sensitivity values for classification of $\mathrm{AB}, \mathrm{CD}$, and $\mathrm{E}$ were $99.04 \%$, $99.43 \%$, and $100 \%$, respectively, while the average specificity values for $\mathrm{AB}, \mathrm{CD}$, and $\mathrm{E}$ were $99.53 \%$, $99.01 \%$, and $100 \%$, respectively. The average improvement given by the proposed method is $2.6 \%$ for accuracy, $2.79 \%, 3.44 \%, 1.25 \%$ for the sensitivity 
Table 3. Classification accuracy (ACC) of epileptic EEG signals on each dataset and scenario

\begin{tabular}{|c|c|c|c|c|c|c|c|c|}
\hline \multirow[t]{2}{*}{ Datasets } & \multirow{2}{*}{$\begin{array}{c}\text { \# Segment } \\
(r)\end{array}$} & \multirow{2}{*}{$\begin{array}{c}\text { Spectrogram } \\
\text { Image } \\
(\mathbf{a} \times \mathbf{x} \text { b) } \\
\end{array}$} & \multicolumn{6}{|c|}{$\operatorname{ACC}(\%)$} \\
\hline & & & Fold-1 & Fold-2 & Fold-3 & Fold-4 & Fold-5 & Average \\
\hline \multirow[t]{7}{*}{ AB-C } & 1 & $129 \times 509$ & 93.33 & 96.67 & 95 & 96.67 & 91.67 & 94.67 \\
\hline & 3 & $129 \times 167$ & 98.33 & 98.33 & 100 & 100 & 96.67 & 98.67 \\
\hline & 5 & $129 \times 99$ & 100 & 98.33 & 100 & 100 & 95 & 98.67 \\
\hline & 7 & $129 \times 70$ & 100 & 98.33 & 100 & 100 & 98.33 & 99.33 \\
\hline & 9 & $129 \times 53$ & 100 & 98.33 & 100 & 100 & 96.67 & 99.00 \\
\hline & 11 & $129 \times 43$ & 100 & 98.33 & 100 & 100 & 98.33 & 99.33 \\
\hline & 13 & $129 \times 36$ & 100 & 98.33 & 100 & 100 & 98.33 & 99.33 \\
\hline \multirow[t]{7}{*}{ AB-D } & 1 & $129 \times 509$ & 98.33 & 96.67 & 100 & 96.67 & 96.67 & 97.67 \\
\hline & 3 & $129 \times 167$ & 100 & 98.33 & 100 & 98.33 & 100 & 99.33 \\
\hline & 5 & $129 \times 99$ & 100 & 100 & 100 & 100 & 100 & 100 \\
\hline & 7 & $129 \times 70$ & 100 & 100 & 100 & 100 & 100 & 100 \\
\hline & 9 & $129 \times 53$ & 100 & 100 & 100 & 100 & 100 & 100 \\
\hline & 11 & $129 \times 43$ & 100 & 100 & 100 & 100 & 100 & 100 \\
\hline & 13 & $129 \times 36$ & 100 & 100 & 100 & 100 & 100 & 100 \\
\hline \multirow[t]{7}{*}{ AB-E } & 1 & $129 \times 509$ & 100 & 95 & 95 & 100 & 96.67 & 97.33 \\
\hline & 3 & $129 \times 167$ & 98.33 & 100 & 98.33 & 100 & 98.33 & 99.00 \\
\hline & 5 & $129 \times 99$ & 100 & 100 & 100 & 100 & 98.33 & 99.67 \\
\hline & 7 & $129 \times 70$ & 100 & 100 & 100 & 100 & 100 & 100 \\
\hline & 9 & $129 \times 53$ & 100 & 100 & 100 & 100 & 100 & 100 \\
\hline & 11 & $129 \times 43$ & 98.33 & 100 & 100 & 100 & 100 & 99.67 \\
\hline & 13 & $129 \times 36$ & 98.33 & 100 & 100 & 100 & 100 & 99.67 \\
\hline \multirow[t]{7}{*}{$\mathrm{AB}-\mathrm{CD}$} & 1 & $129 \times 509$ & 97.75 & 96.25 & 100 & 100 & 92.25 & 97.25 \\
\hline & 3 & $129 \times 167$ & 100 & 98.75 & 100 & 100 & 96.25 & 99.00 \\
\hline & 5 & $129 \times 99$ & 100 & 98.75 & 100 & 100 & 97.50 & 99.25 \\
\hline & 7 & $129 \times 70$ & 100 & 98.75 & 98.75 & 100 & 97.50 & 99.00 \\
\hline & 9 & $129 \times 53$ & 100 & 98.75 & 100 & 100 & 98.75 & 99.50 \\
\hline & 11 & $129 \times 43$ & 100 & 98.75 & 100 & 100 & 98.75 & 99.50 \\
\hline & 13 & $129 \times 36$ & 100 & 98.75 & 98.75 & 100 & 97.50 & 99.00 \\
\hline \multirow[t]{7}{*}{$\mathrm{AB}-\mathrm{CDE}$} & 1 & $129 \times 509$ & 96 & 96 & 97 & 97 & 98 & 96.80 \\
\hline & 3 & $129 \times 167$ & 98 & 99 & 98 & 99 & 100 & 98.80 \\
\hline & 5 & $129 \times 99$ & 98 & 99 & 100 & 99 & 100 & 99.20 \\
\hline & 7 & $129 \times 70$ & 98 & 99 & 100 & 100 & 100 & 99.40 \\
\hline & 9 & $129 \times 53$ & 98 & 100 & 100 & 100 & 100 & 99.60 \\
\hline & 11 & $129 \times 43$ & 99 & 100 & 100 & 100 & 100 & 99.80 \\
\hline & 13 & $129 \times 36$ & 99 & 100 & 100 & 100 & 100 & 99.80 \\
\hline \multirow[t]{7}{*}{ AB-CD-E } & 1 & $129 \times 509$ & 96 & 95 & 100 & 97 & 95 & 96.6 \\
\hline & 3 & $129 \times 167$ & 98 & 97 & 100 & 98 & 100 & 98.6 \\
\hline & 5 & $129 \times 99$ & 99 & 99 & 100 & 98 & 100 & 99.2 \\
\hline & 7 & $129 \times 70$ & 98 & 98 & 100 & 98 & 100 & 98.8 \\
\hline & 9 & $129 \times 53$ & 99 & 99 & 100 & 99 & 100 & 99.4 \\
\hline & 11 & $129 \times 43$ & 98 & 100 & 100 & 98 & 100 & 99.2 \\
\hline & 13 & $129 \times 36$ & 98 & 100 & 100 & 98 & 100 & 99.2 \\
\hline
\end{tabular}


Table 4. Sensitivity(SEN) and specificity (SPE) of epileptic EEG signals classification on each dataset

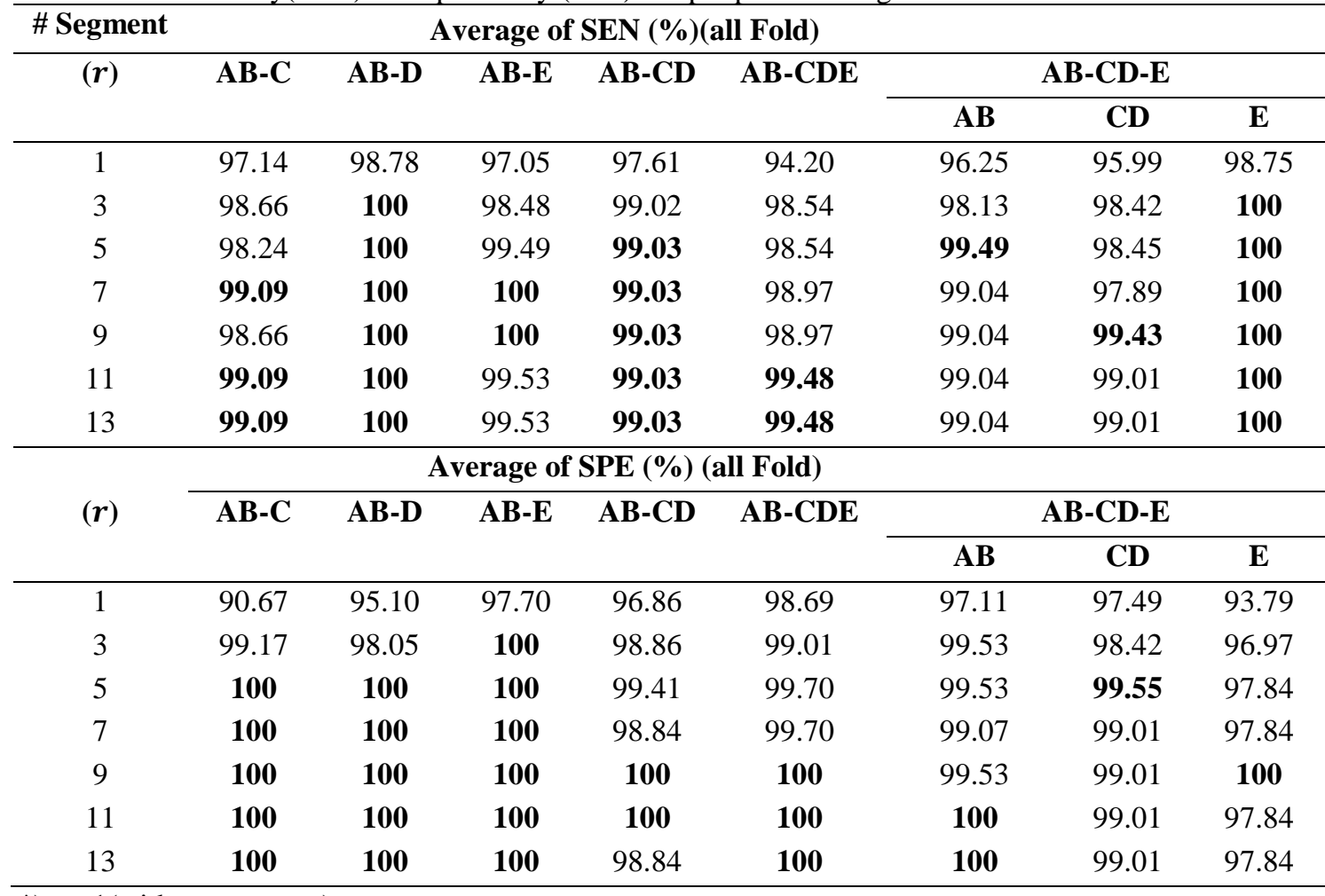

*) $r=1$ (without segment)

of $\mathrm{AB}, \mathrm{CD}, \mathrm{E}$, and $2.42 \%, 1.52 \%, 6.21 \%$ for the specificities of $\mathrm{AB}, \mathrm{CD}, \mathrm{E}$.

From the test results on all datasets, in general, the proposed method by forming a multi-segment signal in the epileptic EEG signal much better performance than the implementation without forming a segment on the original EEG signal. The formation of the EEG signal segment has the effect of multiplying the spectrogram image used in the training process to get the optimal CNN weight and avoid overfitting. The more spectrogram images used in training, it is possible that the more features can be retrieved through a convolutional process with $\mathrm{CNN}$. The ensemble combination process from the results of the signal classification with $\mathrm{CNN}$ on the testing dataset has a role in reducing errors the classification of epileptic EEG signals. Because in the ensemble combination process, the CNN classification results are done by voting as in Eq. (10), so in determining the number of segments must be odd to avoid the same voting value.

\subsection{Discussion}

Many methods have been proposed for the classification of epileptic EEG signals. The EEG signal classification usually includes binary and ternary classification. This study focused on testing several datasets, including AB-C, AB-D, AB-E, AB$\mathrm{CDE}$, and $\mathrm{AB}-\mathrm{CD}-\mathrm{E}$. Comparison of this to the existing methods and the same test dataset is given in Table 5, including Tzallas et al. [54], Orhan et al. [55], Song and Zhang [15], Zhu et al. [56], Hassan and Subasi [57], Tiwari et al. [28], Sharma et al. [30], Gupta et al. [31], Ullah et al. [32], X. Zhao et al. [29], Akut [34], and W. Zhao et al. [33].

Tzallas et al. [54] using time-frequency analysis, and ANN yielded an accuracy of classification for AB-CD-E dataset max $97.72 \%$ and the average of $94.73 \%$. Similar research was also conducted by Orhan et al. [55] by using the K-mean, and multilayer perceptron neural network obtained an accuracy of 95.6\%. Like before, Hasan and Subasi [57] using spectral feature extraction from CEEMDAN mode functions and linear programming boosting spectral classifying with the same dataset obtained an accuracy of 97.6\%. Tiwari and Pachori [28] classified the same dataset using the local binary pattern (LBP), and SVM obtained an accuracy of $98.8 \%$. The same test was also carried out by Sarma et al.[30]. They used IF in the EEG signal to decompose the signal and retrieve its features, including KNNE, LEE, and SE. From the test result using the random forest classifier it obtained a maximum accuracy which is 98\%. Besides, X. Zhao et al. [29] obtained an average of accuracy which is $99.1 \%$ by using Instantaneous energy-based features and BPNN. These researchers use conventional methods, whereas those that use $\mathrm{CNN}$ is as conducted by Ullah et al.[32]. They used 
Table 5. Comparison of the existing methods with the proposed method

\begin{tabular}{|c|l|l|c|}
\hline Reseachers & \multicolumn{1}{|c|}{ Methods } & \multicolumn{1}{|c|}{ Datasets } & ACC (\%) \\
\hline$[54]$ & Time-Frequency Analysis +PCA+ANN & AB-CD-E & 97.72 \\
\hline$[55]$ & K-means +MLPNN & AB-CDE & 98.8 \\
& & AB-CD-E & 95.6 \\
\hline$[15]$ & Wavelet Transform + ELM+ Genetic Algorithm & AB-CDE & 94.2 \\
\hline$[56]$ & Sample Entropy + Multi-Scale K-means & AB-CDE & 99.1 \\
\hline$[57]$ & CEEMDAN + Linear Programming Boosting & AB-CD-E & 97.6 \\
\hline$[28]$ & Local Binary Pattern (LBP) +SVM & AB-E & $\mathbf{1 0 0}$ \\
& & AB-CD-E & 98.8 \\
\hline$[30]$ & KNNE + Random Forest Classifier & AB-CD-E & 98 \\
\hline$[31]$ & Hurst Exponent+ ARMA+SVM & AB-E & 97.27 \\
& & AB-CD & 97.7 \\
\hline$[32]$ & P-1D-CNN & AB-E & 99.7 \\
& & AB-CD & $\mathbf{9 9 . 8}$ \\
& & AB-CDE & $\mathbf{9 9 . 9 5}$ \\
& & AB-CD-E & 99.1 \\
\hline$[29]$ & Instantaneous Energy-Based Features+BPNN & AB-E & $\mathbf{1 0 0}$ \\
& & AB-CD-E & 99.1 \\
\hline$[34]$ & DWT+CNN & AB-CD-E & $\mathbf{9 9 . 4}$ \\
\hline$[33]$ & 1D CNN & AB-E & 99.38 \\
& & AB-CD-E & 96.97 \\
\hline method & Ensemble Combination (Testing) & AB-C & $\mathbf{9 9 . 3 3}$ \\
& & AB-D & $\mathbf{1 0 0}$ \\
& & AB-E & $\mathbf{1 0 0}$ \\
& & AB-CD & 99.5 \\
& & AB-CD-E & 99.8 \\
\hline & & 99.4 \\
\hline
\end{tabular}

$\mathrm{P}-\mathrm{ID}-\mathrm{CNN}$ in the same dataset and yielded an average of accuracy which is $99.1 \%$. Akut [34] used DWT and $\mathrm{CNN}$ for classification and yielded an average of accuracy which is $99.4 \%$. In contrast, W. Zhao et al. [33] obtained an average of accuracy which is $96.97 \%$ by using the $1 \mathrm{D}$ CNN. Using the proposed method on the same dataset, we obtained an average of accuracy which is $99.4 \%$. These results are obtained by dividing the original signal into 9 segments. We only use a CNN model to classify each segment before the ensemble combination process of all the classification results. Compared to conventional methods, our proposed method is better. This occurs because a common problem in conventional methods requires manually setting parameters to get features and does not involve classifiers in selecting important features. Compared to the CNN-based method proposed by Ullah et al. [32] and W. Zhao et al. [33], our proposed method with the same dataset still yielded better results. Ullah et al. [32] and W. Zhao et al. [33] directly input the raw signal/sub-signal on CNN without any specific preprocesses such as transformation to the signal using the time-frequency domain so that there is an important feature of the signal that $\mathrm{CNN}$ cannot yet use for non-binary classification. On the contrary, in our proposed method, the segment/sub-signal is first processed into a spectrogram image (2D) before being forwarded to CNN. Our results for this case are comparable to that of Akut [34] that used DWT (2D) to process the original signal before entering $\mathrm{CNN}$.

For testing, the AB-CDE dataset, among others, was carried out by Orhan et al. [55]. Using K-mean and MLPNN obtained an accuracy of $98.8 \%$. This test was also carried out by Song and Zhang [15] using the wavelet transform, genetic algorithm, and extreme learning machine obtained an accuracy of 94.2\%. Zhu et al. [56] used the entropy sample method and multi-scale K-means, yielded $99.1 \%$ accuracy. Meanwhile, with our proposed method, we obtain an average of accuracy which is $99.8 \%$ when the original signal is divided into 11 and 13 segments. These results confirm that our proposed method has a higher accuracy than the conventional methods. However, it is still lower than the method proposed by Ullah et al. [32], with the average of accuracy which is $99.95 \%$. Although our proposed method's accuracy is not the best in this dataset, the difference between our proposed method's accuracy and the best accuracy is relatively small $(0.15 \%)$.

Testing on the AB-CD dataset, conducted by Gupta et al. [31] and Ullah et al. [32]. Gupta et al. 
[31] used hurst exponent and ARMA parameters as features. Their experiments using SVM obtained an average of accuracy which is $97.7 \%$. Ullah et al. [32], with the proposed method (P-1D-CNN), obtained an average of accuracy which is $99.8 \%$ for this case. Meanwhile, our proposed method obtained an accuracy of $99.5 \%$ when the original signal is divided into 9 and 11 segments. Based on these results, the proposed method is better than the method proposed by Gupta et al. [31] but it is still under Ullah et al. [32]. The method proposed by Gupta has not been running consistently and optimally because the sample size of the dataset is relatively small. We have solved this problem by forming multiple segments of each original signal without overlapping. Our classification accuracy results in this dataset are still lower than Ullah et al. [32] because we only use one CNN model to classify each signal segment before the ensemble process.

Furthermore, for testing the AB-E dataset, the method proposed by Gupta et al. [31] obtained an average of accuracy which is $97.27 \%$, W. Zhao et al. [33] obtained an average of accuracy which is 99.38\%, and Ullah et al. [32] obtained an average of accuracy which is $99.7 \%$. For this case, our proposed method achieved an average of accuracy which is $100 \%$ when the original signal is divided into 7 and 9 segments, so it is better than them. Tiwari et al. [28] and X. Zhao et al. [29], in this case, also yielded an average of accuracy which is $100 \%$. It is interesting in this case that the two CNN-based methods provide average of accuracy under the two conventional methods, although the signal augmentation in the sub-signal has been done. The first reason they made the raw sub-signal as input on $\mathrm{CNN}$ so that there are important features that differentiate the two classes that are not selected by $\mathrm{CNN}$.

For testing by using the AB-C and AB-D datasets, with our proposed method, an average of accuracy which is $99.33 \%$ and $100 \%$ is obtained. For this dataset, we have not found any other methods that carry out testing so we cannot make comparisons yet.

We realize that the comparison of our proposed method with existing methods is far from ideal. The main problem that is difficult for us to avoid is the configuration of datasets for training and testing that may be different. We are currently only able to make comparisons based on the similarity of the testing datasets among other researchers. However, from these comparisons, we can see that our proposed method is comparable.

\section{Conclusion}

We have proposed CNN based on multi-segment EEG signals involving an ensemble combination to classify epileptic EEG signals. This method has been designed by forming a multi-segment on the EEG signal without overlapping and transforming each segment into a spectrogram image (2D) based on the STFT value. Classification of EEG signals is carried out through two classification stages. The first is the classification of each signal segment in each signal channel with one CNN model and the second is the classification using an ensemble combination based on the majority results classification on each segment by CNN. Experiments on several datasets arranged from the Bonn EEG dataset show that our proposed method (with segment) performs better than those without forming a signal segment. The best average of accuracy in the classification is $99.33 \%-100 \%$ for two classes and $99.4 \%$ for three classes. Therefore, the method we propose in this study has great potential to assist neurologists (clinicians) in diagnosing epilepsy patients.

There is still a potential to improve performance in the classification of epilepsy based on EEG signals. In the future, the consideration of overlapping in forming multi-segments to add data to the training process and using several different models in the classification of each segment could improve performance.

\section{Conflicts of Interest}

The authors declare no conflict of interest.

\section{Author Contributions}

Conceptualization, Irwan Budi Santoso, and I Ketut Eddy Purnama; methodology, Irwan Budi Santoso, and I Ketut Eddy Purnama; software, Irwan Budi Santoso; validation, Irwan Budi Santoso, and I Ketut Eddy Purnama; formal analysis, Irwan Budi Santoso, Yudhi Adrianto, Anggraini Dwi Sensusiati, Diah Puspito Wulandari, and I Ketut Eddy Purnama; investigation, I Ketut Eddy Purnama; resources, I Ketut Eddy Purnama; data curation, Irwan Budi Santoso; writing - original draft preparation, Irwan Budi Santoso; writing - review and editing, I Ketut Eddy Purnama, Diah Puspito Wulandari, and Anggraini Dwi Sensusiati; visualization, Irwan Budi Santoso; supervision, I Ketut Eddy Purnama, Diah Puspito Wulandari, and Anggraini Dwi Sensusiati; project administration, I Ketut Eddy Purnama; funding acquisition, I Ketut Eddy Purnama. 


\section{Acknowledgments}

This research is partially funded by Lembaga Pengelola Dana Pendidikan under the scheme of Riset Inovatif Produktif (RISPRO) - Invitasi 2019 Grant, contract number: PRJ-41/LPDP/2019.

\section{References}

[1] World Health Organization, WHO | Epilepsy: $a$ Public Health Imperative. 2019.

[2] I. E. Scheffer, S. Berkovic, G. Capovilla, M. B. Connolly, J. French, L. Guilhoto, E. Hirsch, S. Jain, G. W. Mathern, S. L. Moshe, D. R. Nordli, E. Perucca, T. Tomson, S. Wiebe, Y. H. Zhang, and S. M. Zuberi, "ILAE classification of the epilepsies: position paper of the ILAE Commission for Classification and Terminology", Zeitschrift fur Epileptologie, Vol. 31, No. 4, pp. 296-306, 2017.

[3] F. E. Abd El-Samie, T. N. Alotaiby, M. I. Khalid, S. A. Alshebeili, and S. A. Aldosari, "A Review of EEG and MEG Epileptic Spike Detection Algorithms", IEEE Access, Vol. 6, pp. 6067360688, 2018.

[4] A. F. Hussein, A. N, C. Gomes, A. K. Alzubaidi, Q. A. Habash, L. Santamaria-Granados, J. F. Mendoza-Moreno, and G. Ramirez-Gonzalez, "Focal and Non-Focal Epilepsy Localization: A Review", IEEE Access, Vol. 6, pp. 4930649324, 2018.

[5] S. Siuly, Y. Li, and P. P. Wen, "Clustering technique-based least square support vector machine for EEG signal classification", Computer Methods and Programs in Biomedicine, Vol. 104, No. 3, pp. 358-372, 2011.

[6] S. Siuly and Y. Li, "Designing a robust feature extraction method based on optimum allocation and principal component analysis for epileptic EEG signal classification", Computer Methods and Programs in Biomedicine, Vol. 119, No. 1, pp. 29-42, 2015.

[7] W. Theeranaew, J. McDonald, B. Zonjy, F. Kaffashi, B. D. Moseley, D. Friedman, E. So, J. Tao, M. Nei, P. Ryvlin, R. Surges, R. Thijs, S. Schuele, S. Lhatoo, and K. A. Loparo, "Automated detection of postictal generalized EEG suppression", IEEE Transactions on Biomedical Engineering, Vol. 65, No. 2, pp. 371-377, 2018.

[8] F. Wang, M. Sun, Y. Wang, T. Min, and T. Zang, "Prediction for High Risk Clinical Symptoms of Epilepsy Based on Deep Learning Algorithm", IEEE Access, Vol. 6, pp. 77596-77605, 2018.

[9] H. R. Al Ghayab, Y. Li, S. Siuly, and S. Abdulla,
"Epileptic EEG signal classification using optimum allocation based power spectral density estimation", IET Signal Processing, Vol. 12, No. 6, pp. 738-747, 2018.

[10] R. Djemili, H. Bourouba, and M. C. Amara Korba, "Application of empirical mode decomposition and artificial neural network for the classification of normal and epileptic EEG signals", Biocybernetics and Biomedical Engineering, Vol. 36, No. 1, pp. 285-291, 2016.

[11] Y. Li, X. D. Wang, M. L. Luo, K. Li, X. F. Yang, and Q. Guo, "Epileptic Seizure Classification of EEGs Using Time-Frequency Analysis Based Multiscale Radial Basis Functions", IEEE Journal of Biomedical and Health Informatics, Vol. 22, No. 2, pp. 386-397, 2018.

[12] H. Wang, W. Shi, and C. S. Choy, "Hardware design of real time epileptic seizure detection based on STFT and SVM", IEEE Access, Vol. 6, pp. 67277-67290, 2018.

[13] S. Supriya, S. Siuly, H. Wang, J. Cao, and Y. Zhang, "Weighted Visibility Graph with Complex Network Features in the Detection of Epilepsy", IEEE Access, Vol. 4, pp. 6554-6566, 2016.

[14] R. G. Andrzejak, K. Lehnertz, F. Mormann, C. Rieke, P. David, and C. E. Elger, EEG Time Series Data, Department of Epileptology, Bonn University, 2001. Available online at: http:// epileptologie-bonn.de/cms/front_content.php? idcat $=193 \&$ lang $=3 \&$ changelang $=3$ (accessed on December 5, 2019)

[15] Y. Song and J. Zhang, "Automatic recognition of epileptic EEG patterns via Extreme Learning Machine and multiresolution feature extraction", Expert Systems with Applications, Vol. 40, No. 14, pp. 5477-5489, 2013.

[16] S. Patidar and T. Panigrahi, "Detection of epileptic seizure using Kraskov entropy applied on tunable-Q wavelet transform of EEG signals", Biomedical Signal Processing and Control, Vol. 34, pp. 74-80, 2017.

[17] Z. Li, L. Feng, Y. Wang, C. Wang, and H. Bai, "Hardware design of multiclass SVM classification for epilepsy and epileptic seizure detection", IET Circuits, Devices \& Systems, Vol. 12, No. 1, pp. 108-115, 2017.

[18] N. Nicolaou and J. Georgiou, "Detection of epileptic electroencephalogram based on Permutation Entropy and Support Vector Machines", Expert Systems with Applications, Vol. 39, No. 1, pp. 202-209, 2012.

[19] A A. K. Jaiswal and H. Banka, "Local pattern transformation based feature extraction techniques for classification of epileptic EEG 
signals", Biomedical Signal Processing and Control, Vol. 34, pp. 81-92, 2017.

[20] K. Samiee, P. Kovács, and M. Gabbouj, "Epileptic seizure classification of EEG timeseries using rational discrete short-time fourier transform", IEEE Transactions on Biomedical Engineering, Vol. 62, No. 2, pp. 541-552, 2015.

[21] Ö. F. Alçin, S. Siuly, V. Bajaj, Y. Guo, A. Şengur, and Y. Zhang, "Multi-category EEG signal classification developing time-frequency texture features based Fisher Vector encoding method", Neurocomputing, Vol. 218, pp. 251258, 2016.

[22] H. R. Al Ghayab, Y. Li, S. Abdulla, M. Diykh, and X. Wan, "Classification of epileptic EEG signals based on simple random sampling and sequential feature selection", Brain Informatics, Vol. 3, No. 2, pp. 85-91, 2016.

[23] O. Yildirim, M. Talo, B. Ay, U. B. Baloglu, G. Aydin, and U. R. Acharya, "Automated detection of diabetic subject using pre-trained 2D-CNN models with frequency spectrum images extracted from heart rate signals", Computers in Biology and Medicine, Vol. 113, No. June, p. 103387, 2019.

[24] E. Alhadhrami, M. Al-Mufti, B. Taha, and N. Werghi, "Learned Micro-Doppler Representations for Targets Classification Based on Spectrogram Images", IEEE Access, Vol. 7, pp. 139377-139387, 2019.

[25] Y. Zeng, M. Zhang, F. Han, Y. Gong, and J. Zhang, "Spectrum Analysis and Convolutional Neural Network for Automatic Modulation Recognition", IEEE Wireless Communications Letters, Vol. 8, No. 3, pp. 929-932, 2019.

[26] L. Yuan and J. Cao, "Patients' EEG Data Analysis via Spectrogram Image", In: Proc. of International Conf. on Intelligent Decision Technologies, pp. 13-21, 2018.

[27] G. Xu, X. Shen, S. Chen, Y. Zong, C. Zhang, H. Yue, M. Liu, F. Chen, and W. Che, "A Deep Transfer Convolutional Neural Network Framework for EEG Signal Classification", IEEE Access, Vol. 7, pp. 112767-112776, 2019.

[28] A. K. Tiwari, R. B. Pachori, V. Kanhangad, and B. K. Panigrahi, "Automated Diagnosis of Epilepsy Using Key-Point-Based Local Binary Pattern of EEG Signals", IEEE Journal of Biomedical and Health Informatics, Vol. 21, No. 4, pp. 888-896, 2017.

[29] X. Zhao, R. Zhang, Z. Mei, C. Chen, and W. Chen, "Identification of epileptic seizures by characterizing instantaneous energy behavior of EEG”, IEEE Access, Vol. 7, pp. 70059-70076, 2019.
[30] R. R. Sharma, P. Varshney, R. B. Pachori, and S. K. Vishvakarma, "Automated System for Epileptic EEG Detection Using Iterative Filtering", IEEE Sensors Letters, Vol. 2, No. 4, pp. 1-4, 2018.

[31] A. Gupta, S. Member, P. Singh, and M. Karlekar, "A Novel Signal Modeling Approach for Classification of Seizure and Seizure-free EEG Signals", IEEE Transactions on Neural Systems and Rehabilitation Engineering, Vol. 4320, No. c, pp. 1-10, 2018.

[32] I. Ullah, M. Hussain, E. ul H. Qazi, and H. Aboalsamh, "An automated system for epilepsy detection using EEG brain signals based on deep learning approach", Expert Systems with Applications, Vol. 107, pp. 61-71, 2018.

[33] W. Zhao, W. Zhao, W. Wang, X. Jiang, X. Zhang, Y. Peng, B.Zhang, and G. Zhang, "A Novel Deep Neural Network for Robust Detection of Seizures Using EEG Signals", Computational and Mathematical Methods in Medicine, Vol. 2020, 2020.

[34] R. Akut, "Wavelet based deep learning approach for epilepsy detection", Health Information Science and Systems, Vol. 7, No. 1, pp. 1-9, 2019.

[35] Ö. Türk and M. S. Özerdem, "Epilepsy detection by using scalogram based convolutional neural network from eeg signals", Brain Sciences, Vol. 9, No. 5, 2019.

[36] R. G. Andrzejak, K. Lehnertz, F. Mormann, C. Rieke, P. David, and C. E. Elger, "Indications of nonlinear deterministic and finite-dimensional structures in time series of brain electrical activity: Dependence on recording region and brain state", Physical Review E, Vol. 64, No. 6, p. 061907, 2001.

[37] Siuly and Y. Li, "A novel statistical algorithm for multiclass EEG signal classification", Engineering Applications of Artificial Intelligence, Vol. 34, pp. 154-167, 2014.

[38] A. Prieto-Guerrero and G. Espinosa-Paredes, Linear Signal Processing Methods and Decay Ratio Estimation, Elsevier Ltd, 2019.

[39] N. Kehtarnavaz, "Frequency Domain Processing", Digital Signal Processing System Design, Vol. 1, pp. 175-196, 2008.

[40] A. V. Oppenheim, R. W. Schafer, and J. R. Buck, Discrete-Time Signal Processing, pp. 468-471, Prentice Hall, New Jersey, 1999.

[41] D. Lai, X. Zhang, K. Ma, Z. Chen, W. Chen, H. Zhang, H. Yuan, and L. Ding, "Automated Detection of High Frequency Oscillations in Intracranial EEG Using the Combination of Short-Time Energy and Convolutional Neural 
Networks", IEEE Access, Vol. 7, pp. 8250182511, 2019.

[42] S. Ren, K. He, R. Girshick, and J. Sun, "Faster R-CNN: Towards Real-Time Object Detection with Region Proposal Networks", IEEE Transactions on Pattern Analysis and Machine Intelligence, Vol. 39, No. 6, pp. 1137-1149, 2017.

[43] A.Krizhevsky, I. Sutskever and G. E. Hinton, "Convolutional Neural Networks ImageNet Classification with Deep", Communication of the ACM, Vol. 60, No. JUNE, pp. 84-90, 2017.

[44] K. He, X. Zhang, S. Ren, and J. Sun, "Delving deep into rectifiers: Surpassing human-level performance on imagenet classification", In: Proc. of the IEEE International Conf. on Computer Vision, Vol. 2015 Inter, pp. 10261034, 2015.

[45] Y. Yuan, G. Xun, K. Jia, and A. Zhang, "A multi-view deep learning framework for EEG seizure detection", IEEE Journal of Biomedical and Health Informatics, Vol. 23, No. 1, pp. 8394, 2019.

[46] X. Glorot and Y. Bengio, "Understanding the difficulty of training deep feedforward neural networks", In: Proc. of International Conf. on Artificial Intelligence and Statistics (AISTATS), Vol. 9, pp. 249-256, 2010.

[47] V. Dumoulin and F. Visin, "A guide to convolution arithmetic for deep learning", arXiv:1603.07285v2, pp. 1-31, 2018.

[48] S. S. Ioffe and C. Szegedy, "Batch normalization: Accelerating deep network training by reducing internal covariate shift", In: Proc. of 32nd International Conf. on Machine Learning, ICML 2015, Vol. 1, pp. 448-456, 2015.

[49] J. Nagi, F. Ducatelle, G. A. Di Caro, D. Ciresan, U. Meier, A. Giusti, F. Nagi, J. Schmidhuber, and L. M. Gambardella, "Max-pooling convolutional neural networks for vision-based hand gesture recognition", In: Proc. of 2011 IEEE International Conf. on Signal and Image Processing Applications, ICSIPA 2011, pp. 342347, 2011.

[50] C. M. Bishop, Pattern Recognition and Machine Learning, Springer, New York, NY.2006.

[51] C. Zhang and Y. Ma, Ensemble machine learning: Methods and applications, Springer, New York Dordrecht Heidelberg London, 2012.

[52] A. Pe and J. A. Lozano, "Sensitivity Analysis of $\mathrm{k}$-Fold Cross Validation in Prediction Error Estimation", IEEE Transactions on Pattern Analysis and Machine Intelligence, Vol. 32, No. 3, pp. 569-575, 2010.
[53] Y. Jiao and P. Du, "Performance measures in evaluating machine learning based bioinformatics predictors for classifications", Quantitative Biology, Vol. 4, No. 4, pp. 320-330, 2016.

[54] A. T. Tzallas, M. G. Tsipouras, and D. I. Fotiadis, "Automatic Seizure Detection Based on TimeFrequency Analysis and Artificial Neural Networks", Computational Intelligence and Neuroscience, Vol. 2007, pp. 1-13, 2007.

[55] U. Orhan, M. Hekim, and M. Ozer, "EEG signals classification using the K-means clustering and a multilayer perceptron neural network model", Expert Systems with Applications, Vol. 38, No. 10, pp. 13475-13481, 2011.

[56] G. Zhu, Y. Li, P. Wen, S. Wang, and N. Zhong, "Unsupervised classification of epileptic EEG signals with multi scale K-means algorithm", Lecture Notes in Computer Science (including subseries Lecture Notes in Artificial Intelligence and Lecture Notes in Bioinformatics), Vol. 8211 LNAI, pp. 158-167, 2013.

[57] A. R. Hassan and A. Subasi, "Automatic identification of epileptic seizures from EEG signals using linear programming boosting", Computer Methods and Programs in Biomedicine, Vol. 136, pp. 65-77, 2016. 